\title{
Fibrinolysis Resistance: A Potential Mechanism Underlying COVID-19 Coagulopathy
}

\author{
Emmanuel Weiss ${ }^{1,2,3}$ Olivier Roux ${ }^{4}$ Jean-Denis Moyer ${ }^{1}$ Catherine Paugam-Burtz ${ }^{1,2}$ \\ Larbi Boudaoud $^{5}$ Nadine Ajzenberg ${ }^{6,7}$ Dorothée Faille ${ }^{6,7}$ Emmanuelle de Raucourt ${ }^{5,7}$
}

${ }^{1}$ Department of Anesthesiology and Critical Care, Beaujon Hospital, DMU Parabol, AP-HP.Nord, Paris, France

2 Inserm UMR_S1149, Inserm et Université de Paris, Paris, France

${ }^{3}$ EASL CLIF Consortium, European Foundation for the Study of Chronic Liver Failure, EF CLIF, Barcelona, Spain

${ }^{4}$ Hepatology \& Liver Intensive Care Unit, Hôpital Beaujon, Paris, France

${ }^{5}$ Service Hématologie Biologique, Hôpital Beaujon, Paris, France

${ }^{6}$ Laboratoire d'hématologie, APHP, Hôpital Bichat, Paris, France

${ }^{7}$ UMR_S1148, Inserm et Université de Paris, Paris, France

Thromb Haemost 2020;120:1343-1345.

We read with great interest the article by Spiezia et al, published in a recent issue of the journal, showing a severe hypercoagulability on thromboelastometry profiles of 22 critically ill patients with novel coronavirus (SARS-CoV-2). ${ }^{1}$ These results are in agreement with a growing body of evidence suggesting that COVID-19 severe infection predisposes to venous thromboembolism and even primary pulmonary thrombosis, ${ }^{2}$ and that abnormal coagulation parameters are associated with poor prognosis. $^{3}$ The thrombotic risks in COVID-19 are such that consensus guidance has been issued for thromboprophylaxis, ${ }^{3-5}$ leading to a large prescription of high doses of low molecular weight (LMWH) or unfractionated heparin, notwithstanding the need for more evidence. Tang et al reported high rates of overt disseminated intravascular coagulation according to the International Society on Thrombosis and Haemostasis diagnostic criteria. ${ }^{6}$ Conversely, in the study from Spiezia et al, standard tests and thromboelastography profiles suggested, rather than a consumptive coagulopathy, a severe hypercoagulability linked to hyperfibrinogenemia. ${ }^{1}$

Here, we highlight that resistance to fibrinolysis could be an additional underlying mechanism to the hypercoagulable state that predisposes COVID-19 patients to thrombosis. Using thromboelastometry (ROTEM, Werfen), we investigated the profiles of tissue plasminogen activator (tPA)-induced fibrinolysis in five patients admitted to the intensive care unit (ICU) of Beaujon University Hospital between April 21 and May 4, 2020 for COVID-19-related acute respiratory distress syndrome (ARDS). Thromboelastometry was performed in the presence and absence of recombinant tPA (r-tPA, $80 \mathrm{ng} / \mathrm{mL})$ in these five patients and compared with five healthy controls. ${ }^{7}$ The patients

received

May 13, 2020

accepted after revision

May 28, 2020

Address for correspondence Emmanuel Weiss, MD, PhD, Department of Anesthesiology and Critical Care, Beaujon University Hospital, 100 bld du general Leclerc, F92110, Clichy, France

(e-mail: emmanuel.weiss@aphp.fr).

provided written informed consent when possible. Alternatively, informed consent was given orally by next-of-kin because they could not visit the ICU in the context of pandemia. Healthy controls were hospital employee volunteers involved in the research. All patients were males, mean ( \pm standard deviation) age and Sequential Organ Failure Assessment scores were $57 \pm 15$ years and $9 \pm 2$, respectively. All patients had hyperfibrinogenemia (mean fibrinogen $7.4 \pm 2.4 \mathrm{~g} / \mathrm{L}$ ) except one. Other laboratory data were: hemoglobin: $10.1 \pm 1.6 \mathrm{~g} / \mathrm{dL}$, platelet count: $440 \pm 27010^{9} / \mathrm{L}$, factor V: $177 \pm 37 \%$, and D-dimer: $1975 \pm 1623 \mathrm{ng} / \mathrm{mL}$. As recommended by the GFHT/GIHP, ${ }^{4}$ patients received LMWH with dosage tailored to body mass index and to the risk of thrombosis evaluated using daily Ddimer and fibrinogen measurements. Despite this thromboprophylaxis, three out of our five patients developed an acute pulmonary embolism during the follow-up.

As reported by Spiezia et al, ${ }^{1}$ thromboelastometry profiles showed a hypercoagulable state in COVID-19 patients: maximum clot firmness (MCF) was elevated in the EXTEM: $77 \pm 5 \mathrm{~mm}$ (normal range 50-72) and FIBTEM: $46 \pm 18 \mathrm{~mm}$ (normal range 9-25 mm) assays in the four patients with hyperfibrinogenemia but was normal in patient 5 who exhibited normal fibrinogen level (EXTEM: $57 \mathrm{~mm}$ and FIBTEM: $20 \mathrm{~mm}$ ). In addition, as shown in - Fig. 1, when adding r-tPA to the ROTEM assay, clot lysis was observed after 60 minutes in all controls but not in patients. Maximum lysis (ML) ranged from 85 to $100 \%$ of MCF in controls ( $94 \pm 6.3$ ) and from 4 to $20 \%$ $(12 \pm 6)$ in patients, showing resistance to fibrinolysis in the latter group. Resistance to r-tPA-induced fibrinolysis could be related to high fibrinogen level; however, patient 5 with normal

(c) 2020 Georg Thieme Verlag KG Stuttgart · New York
DOI https://doi.org/ 10.1055/s-0040-1713637. ISSN 0340-6245. 
EXTEM Healthy controls
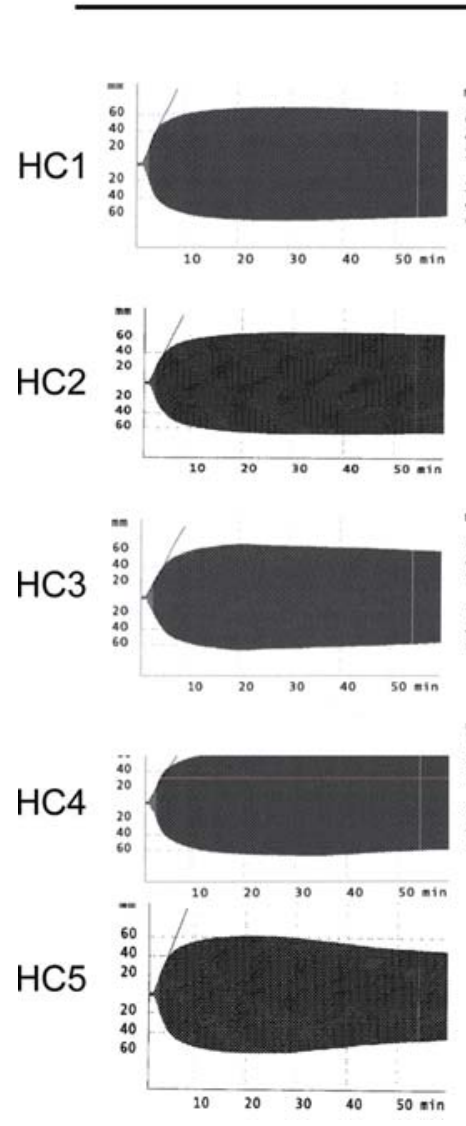
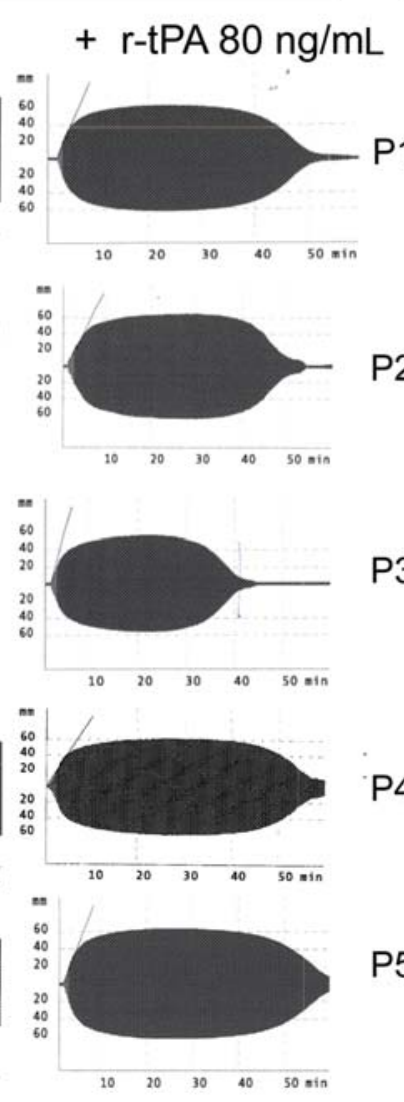

EXTEM Patients with COVID-19
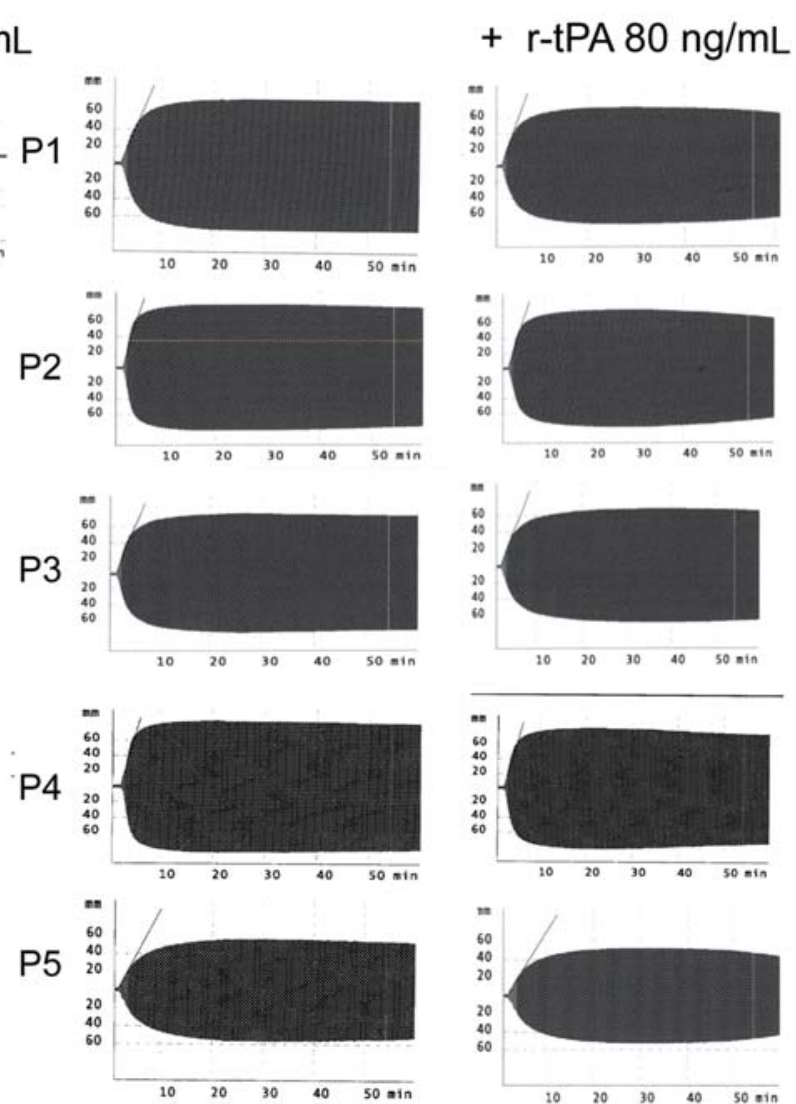

Fig. 1 Thromboelastometry profiles of patients with COVID-19 and of healthy controls in the presence and absence of recombinant tissue plasminogen activator ( $r$-tPA). HC, healthy control; P, patient.

fibrinogen level also exhibited an impaired clot lysis at 60 minutes (ML: 20\%). Furthermore, clot lysis was evaluated in the same conditions in a patient with inflammatory bowel disease (negative for COVID-19) with fibrinogen level at $6 \mathrm{~g} / \mathrm{dL}$. High MCF in EXTEM assay $(75 \mathrm{~mm})$ was observed but the fibrinolysis at 60 minutes was comparable to controls with a ML at 76\% (data not shown). These results suggest that fibrinolytic system is dysregulated in patients admitted with ARDS due to COVID-19 pneumonia. Unfortunately, the main actors of the fibrinolytic pathway, especially plasminogen activator inhibitor 1 (PAI-1) and tPA, could not be evaluated in these five patients and controls. Further studies including measurement of fibrinolytic components and other tests such as euglobulin clot lysis time, should be performed in a larger number of patients to confirm these preliminary data. However, a resistance to t-PA-induced fibrinolysis was already reported in patients with ARDS, who exhibited elevated PAI-1 levels. ${ }^{8}$ In these patients, fibrin deposits are found in the lungs due to dysregulation of the coagulation and fibrinolytic systems. ${ }^{8}$ These observations suggest that r-tPA could be considered, as a therapeutic approach for COVID-19 in a selected group of patients. In those patients, heparin therapy should be carefully monitored and the risk of bleeding should be assessed using, for instance, the HAS-BLED score. ${ }^{8,9}$ Oxygenation improvements after r-tPA treatment were recently reported in three patients with COVID-19 and ARDS. ${ }^{10}$ How- ever, these observed improvements were only transient, suggesting that the optimal dosing regimen may be higher than usual in COVID-19 and that, in accordance with our own data, response to r-tPA is impaired in COVID-19 patients.

Here, we provide preliminary results suggesting that, in addition to hyperfibrinogenemia-related hypercoagulability as reported by Spiezia et $\mathrm{al}^{1}{ }^{1}$ impaired fibrinolysis could contribute to the severe coagulopathy observed in these patients. Larger prospective studies evaluating the fibrinolytic pathways in patients with COVID-19 and ARDS are needed to confirm these results to better understand disease mechanisms and help define new therapeutic approaches.

\section{Authors' Contributions}

E.W. participated in research design, in the writing of the paper, in the performance of the research, and in data analysis. O.R. participated in research design and in the writing of the paper. J.-D.M. participated in research design and in the writing of the paper. C.P.-B. participated in the writing of the paper. L.B. participated in the performance of the research and in data analysis. N.A. participated in research design, in the performance of the research, and in data analysis. D.F. participated in the performance of the research in data analysis, and in the writing of the paper. E.d. R. participated in research design, in the writing of the paper, in the performance of the research, and in data analysis. 


\section{Funding}

None.

\section{Conflict of Interest}

E.W. reports personal fees from MSD France and Biomerieux and travel reimbursements from MSD France. The other authors report no conflict of interest related to this work.

\section{References}

1 Spiezia L, Boscolo A, Poletto F, et al. COVID-19-related severe hypercoagulability in patients admitted to intensive care unit for acute respiratory failure. Thromb Haemost 2020;120(06):998-1000

2 Cattaneo M, Bertinato EM, Birocchi S, et al. Pulmonary embolism or pulmonary thrombosis in COVID-19? Is the recommendation to use high-dose heparin for thromboprophylaxis justified? Thromb Haemost 2020. Doi: 10.1055/s-0040-1712097

3 Bikdeli B, Madhavan MV, Jimenez D, et al. CoVID-19 and thrombotic or thromboembolic disease: implications for prevention, antithrombotic therapy, and follow-up. J Am Coll Cardiol 2020. Doi: 10.1016/j.jacc.2020.04.031

4 Susen S, Tacquard CA, Godon A, et al. Traitement anticoagulant pour la prévention du risque thrombotique chez un patient hospitalisé avec COVID-19 et surveillance de l'hémostase. Propositions du GIHP et du GFHT. 2020. Available at: https://sfar.org/download/traitement-anticoagulant-pour-la-prevention-du- risque-thrombotique-chez-un-patient-hospitalise-avec-covid-19 -et-surveillance-de-lhemostase/?wpdmdl=25834\&refresh $=5$ ec 8 de624218d1590222434. Accessed June 2, 2020

5 Zhai Z, Li C, Chen Y, et al; Prevention Treatment of VTE Associated with COVID-19 Infection Consensus Statement Group. Prevention and treatment of venous thromboembolism associated with coronavirus disease 2019 infection: a consensus statement before guidelines. Thromb Haemost 2020;120(06):937-948

6 Tang N, Li D, Wang X, Sun Z. Abnormal coagulation parameters are associated with poor prognosis in patients with novel coronavirus pneumonia. J Thromb Haemost 2020;18(04): $844-847$

7 Ghebouli R, Loyau S, Maire M, et al. Amino-fucoidan as a vector for rtPA-induced fibrinolysis in experimental thrombotic events. Thromb Haemost 2018;118(01):42-53

8 Whyte CS, Morrow GB, Mitchell JL, Chowdary P, Mutch NJ. Fibrinolytic abnormalities in acute respiratory distress syndrome (ARDS) and versatility of thrombolytic drugs to treat COVID-19. J Thromb Haemost 2020. Doi: 10.1111/jth.14872

9 Pisters R, Lane DA, Nieuwlaat R, de Vos CB, Crijns HJ, Lip GY. A novel user-friendly score (HAS-BLED) to assess 1-year risk of major bleeding in patients with atrial fibrillation: the Euro Heart Survey. Chest 2010;138(05):1093-1100

10 Wang J, Hajizadeh N, Moore EE, et al. Tissue plasminogen activator (tPA) treatment for COVID-19 associated acute respiratory distress syndrome (ARDS): a case series. J Thromb Haemost 2020. Doi: $10.1111 /$ jth. 14828 